\title{
Fractal analysis of the large-scale stellar mass distribution in the Sloan Digital Sky Survey
}

\author{
José Gaite \\ Applied Physics Dept., ETSIAE, Univ. Politécnica de Madrid, E-28040 Madrid, Spain \\ E-mail: jose.gaite@upm.es
}

\begin{abstract}
A novel fractal analysis of the cosmic web structure is carried out, employing the Sloan Digital Sky Survey, data release 7. We consider the large-scale stellar mass distribution, unlike other analyses, and determine its multifractal geometry, which is compared with the geometry of the cosmic web generated by cosmological $N$-body simulations. We find a good concordance, the common features being: (i) a minimum singularity strength $\alpha_{\min }=1$, which corresponds to the edge of diverging gravitational energy and differs from the adhesion model prediction; (ii) a "supercluster set" of relatively high dimension where the mass concentrates; and (iii) a non-lacunar structure, like the one generated by the adhesion model.
\end{abstract}

Keywords: cosmic web, galaxy clusters, redshift surveys, superclusters

\section{Contents}

1 Introduction $\quad 2$

2 Samples of galaxies from the SDSS DR7 3

2.1 Volume-limited samples of galaxies 4

2.2 Choice of coordinates 4

3 Multifractal analysis $\quad 5$

3.1 Errors in multifractal analysis 8

3.2 Multifractal spectrum from cosmological $N$-body simulations 9

4 Procedure and Results $\quad 11$

4.1 Selection of volume-limited samples 11

4.2 Homogeneity scale 12

$\begin{array}{ll}4.3 \text { Values of } v_{0} \text { for our volume-limited samples } & 13\end{array}$

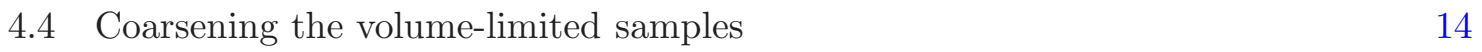

$\begin{array}{lll}4.5 & \text { Multifractal spectrum } & 14\end{array}$

5 Conclusions and discussion $\quad 17$ 


\section{Introduction}

The large scale structure of the universe can be described as a "cosmic web," with characteristic though irregular geometric features that extend over lengths of tens of megaparsecs. On larger scales, the isotropy and homogeneity of the universe gradually manifest themselves, in accord with the Cosmological Principle. The cosmic web structure can be generated by models of the cosmic gravitational dynamics, namely, the Zeldovich approximation and the adhesion model $[1,2]$ and has been found in galaxy surveys $[3,4]$ and cosmological $N$-body simulations [5-7]. The structure actually consists of a web of filaments and sheets of multiple sizes, which represent the patterns of gravitational clustering of matter. This type of geometric structure, with features on ever decreasing scales, belongs in the domain of fractal geometry. Of course, the geometric features of the real cosmic-web have sizes that are bounded below by some scale determined by non-gravitational dynamics (and above by the homogeneity scale).

Fractal models of the universe arose from the idea of a hierarchy of galaxy clusters that continues indefinitely towards the largest scales [8], an idea that originated a debate about the scale of transition to homogeneity [9-14]. Many fractal analyses of galaxy clustering have been motivated or influenced by this debate, but the fractal analysis of the large-scale structure of the universe is interesting in its own right. Early fractal analyses and, specifically, multifractal analyses comprise analyses of the distribution of galaxies [15-17] and also of the distribution of dark matter in cosmological $N$-body simulations [18-20]. Recent $N$-body simulations have better resolution and their analysis reveals new fractal aspects of the cosmic web [21-26]. However, the combined studies of galaxy surveys and $N$-body simulations have not led to a full description of the fractal geometry of the cosmic web and, in particular, to a definite relation between the geometries of the distributions of galaxies and of dark matter. For example, it is not clear that the much studied power-law dependence of the galaxy-galaxy correlation function coincides with an analogous dependence of the correlation function of the dark matter distribution. It is not even clear that these two correlation functions can be directly related, because individual galaxies are not like dark matter particles.

Galaxies are visible because of their baryonic content, and the combined dynamics of cold dark matter and baryonic gas has also been the object of simulations of large-scale structure formation. The comparison between the fractal features of the baryonic gas and dark matter distributions in the result of one of these simulations shows that they are essentially equal [24]. This suggests that a direct comparison of the fractal features of the distributions of observed visible mass and of simulated dark matter should show good concordance.

The study of galaxy clustering by means of correlation functions considers galaxies as equivalent point-like particles [10], much like the particles of dark matter or gas of cosmological $N$-body simulations. Of course, real galaxies are not point like and their spatial extensions are considerable, and even larger than their visible components (even considering only the baryonic part). Nor are galaxies equivalent to one another. Galaxy catalogs provide us with their locations as point-like particles and also with some characteristics that actually distinguish them but normally do not provide us with their masses. The neglect of galaxy masses in the analysis of the large-scale structure is equivalent to assigning the same mass to all galaxies, which is a questionable approximation. Pietronero [15] already noticed the broad range of known galaxy masses and argued that it makes the properties of the spatial mass distribution substantially more complex, to such extent that a multifractal analysis is necessary, instead of the calculation of correlations of galaxy positions. However, in absence of galaxy masses in the catalogs, the multifractal analysis that has been usually performed 
only considers the number density of galaxies $[11,14]$. A notable exception is the early work of Pietronero and collaborators in which they calculated the masses of galaxies from the observed luminosities by assuming a simple mass-luminosity relation (a power law) and thence carried out a proper multifractal analysis [9, 12].

That work of Pietronero et al as well as the contemporary multifractal analyses of other researchers that did not take galaxy masses into account were limited by the galaxy catalogs then available. Fortunately, we have now available better catalogs, which contain, in particular, good estimates of stellar masses of galaxies, obtained with sophisticated methods $[27,28]$. These masses can be used to achieve a more realistic description of the distribution of visible mass. In fact, a quantitative comparison between the statistical properties of the distributions of matter in galaxy surveys and of gas or dark matter in $N$-body simulations requires us to take galaxy masses into account. In contrast, the spatial extensions of galaxies are hardly relevant for the study of the large-scale structure.

We analyze in this work the galaxy distribution in the Sloan Digital Sky Survey, data release 7 (SDSS-DR7), employing the New York University Value-Added Galaxy Catalog (NYU-VAGC) [29] and taking into account the stellar mass content of galaxies. We restrict ourselves to the statistical and geometric properties of the cosmic web that can be determined by a multifractal analysis (for a morphological analysis of the supercluster-void network in SDSS-DR7, see Ref. [30]). Previous studies of the distribution of SDSS galaxies in redshift space are mainly concerned with the problem of the transition to homogeneity, namely, the transition from middle-scale power-law correlations to very-large-scale uniformity [31-34]. These studies do not consider the galaxy masses and look for homogeneity in the number density of galaxies. The scale of homogeneity will feature in our multifractal analysis, as a relevant parameter, but we focus on the properties of the multifractal spectrum and its comparison with the multifractal spectrum found in $N$-body simulations of the Lambda cold dark matter (LCDM) model, with or without gas. At any rate, we will try to compare our results with previous results, especially, with the results of Verevkin et al [33] and ChacónCardona et al [34], who also study the SDSS-DR7. We also compare our analysis with multifractal analyses of older catalogs.

To summarize this work, we first describe the SDSS data employed and the definition of volume-limited samples (Sect. 2). Next, we describe the details of our method of multifractal analysis, including examples of its application to $N$-body simulations (Sect. 3). The results of the analysis of three volume-limited samples of the SDSS, especially, the stellar-mass distribution multifractal spectrum, are contained in Sect. 4, including a comparison with the results of cosmological $N$-body simulations. Finally, we present our conclusions and discuss them in Sect. 5.

\section{Samples of galaxies from the SDSS DR7}

The Sloan Digital Sky Survey in its seventh data release [35] covers one quarter of the sky and has information about galaxies, quasars and stars. The galaxy data have been improved and included in the The New York U. Value-Added Galaxy Catalog (NYU-VAGC) by the research group of M.R. Blanton and D.W. Hogg [29]. Two cuts in apparent magnitude are made in the Petrosian r spectral band, located at $6165 \AA$ : the upper cut, which has to be present in every survey, indicates the faintest objects that can be detected, while the lower cut is made to prevent contamination by very bright objects. Following Ref. [30], we take the lower cut at apparent magnitude $m_{\mathrm{r}}=12.5$ and use the range $12.5<m_{\mathrm{r}}<17.77$. 
The SDSS-DR7 galaxy redshifts extend from nearly null redshift to $z \simeq 0.4$. We choose for our initial sample the following redshift limits: $z>0.001$, to exclude galaxies with considerable peculiar velocities, and $z<0.1$, because we do not need the sample to be deep. Indeed, SDSS galaxies with $z>0.1$ are more luminous and their number densities are considerably smaller, as shown by the luminosity function of SDSS galaxies [36, Fig. 8]. Therefore, the galaxies with $z>0.1$ of the SDSS-DR7 are less suitable for obtaining information on the smaller scales. We further restrict our initial sample to the SDSS main angular area (see Sect. 2.2). The number of galaxies in the sample is 305854 .

\subsection{Volume-limited samples of galaxies}

As in any redshift survey with limits in apparent magnitude, the mean number density of SDSS-DR7 galaxies decreases with redshift. It is necessary to construct volume-limited subsamples of the full sample to correct this radial selection effect $[12,14]$. Unlike in Refs. [3134], in which volume-limited subsamples of galaxies are defined by their ranges of absolute magnitudes, here they are defined by redshift ranges, which determine the corresponding absolute magnitude ranges. The cuts in absolute magnitude are given by the expression of the absolute magnitude in terms of apparent magnitude and redshift [10]:

$$
M_{\mathrm{abs}}=m_{\mathrm{r}}-5 \log _{10} R(z)-25-K(z),
$$

where $m_{\mathrm{r}}$ is the apparent magnitude in the Petrosian $\mathrm{r}$ band, $R$ is the luminosity distance in Mpc, and $K(z)$ is the k-correction for the SDSS r band. In Refs. [31, 33, 34], two different approximations for the calculation of the k-correction have been employed. Here we employ the approximation of Chilingarian et al [37], which is appropriate in our case.

Since we construct volume-limited (VL) samples by specifying their ranges of redshift and the redshifts are associated to individual galaxies, it is easy to analyze how a VL sample changes when $R(z)$ or $K(z)$ change, for example, after a change of the cosmological model. In this regard, we assume a standard LCDM cosmology, with $\Omega_{\mathrm{m}}=0.3, \Omega_{\Lambda}=0.7$, but we have checked that changes in the parameters within reasonable ranges do not alter the results. For the Hubble constant, we may think of the choice $h=1$, but our VL samples, in terms of ranges of $z$, are independent of $h$, because their construction only involves ratios of pairs of values of $R(z)$. In consonance, distances will be expressed in $\mathrm{Mpc} / h$.

Before deciding the VL samples to employ, it is convenient to explain our choice of coordinates, with the angular selection, and also consider the requirements of our multifractal analysis. The selection of our VL samples is described in Sect. 4.1.

\subsection{Choice of coordinates}

Let us consider first the angular coordinates for fixed radial distance. The most convenient angular coordinate systems are orthogonal systems such that they preserve the area, that is to say, such that the element of area is just the product of the line elements along the two coordinates, like in Cartesian coordinates. This type of coordinates is common in geography [38] and have been employed for multifractal analysis of $N$-body simulated halos in Ref. [26]. Equal area coordinates can be defined in terms of angular spherical coordinates: one coordinate is just the longitude and the other is the sine of the latitude. The equatorial coordinates, namely, right ascension $\alpha$ and declination $\delta$, are indeed spherical coordinates and could be used for this purpose. However, the SDSS imaging camera scans the sky in strips along particular great circles, so that the appropriate coordinate system, called the survey coordinate system in Ref. [39], is a different system of angular spherical coordinates, 

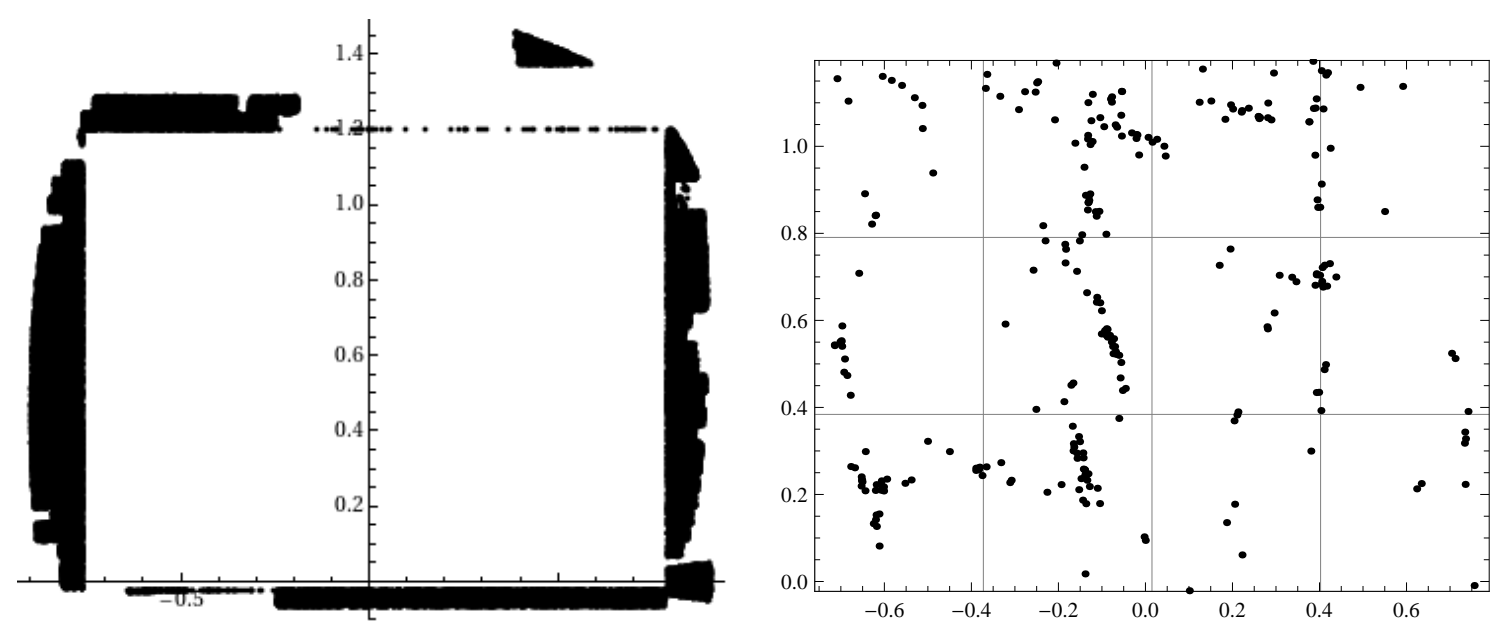

Figure 1. Our system of equal-area angular coordinates $s l$ and $f$ and the selected rectangle $[-0.76,0.79] \times[0.02,1.20]:$ (Left) The small portion of SDSS-DR7 main-area galaxies left out of the rectangle; (right) radial slice $(0.01<z<0.0107)$ of the galaxy distribution in redshift space, showing the cosmic web structure.

with poles at $\alpha=95^{\circ}, \delta=0^{\circ}$, and $\alpha=275^{\circ}, \delta=0^{\circ}$. The latitude and longitude measured from these poles are called $\lambda$ and $\eta$, respectively. In Ref. [39], the origin of $\eta$ is set to the point $\alpha=185^{\circ}, \delta=32.5^{\circ}$ but we have set it to $\alpha=185^{\circ}, \delta=0^{\circ}$, that is to say, the middle point of the semicircle $\delta=0^{\circ}, \alpha \in\left[95^{\circ}, 275^{\circ}\right]$. Therefore, our equal-area coordinates are $s l=\sin \lambda$ and $f=\eta+32.5^{\circ}\left(\pi / 180^{\circ}\right)$ (in radians). They are related to $\alpha$ and $\delta$ by the transformations

$$
\begin{aligned}
s l & =\sin (\alpha-185 \pi / 180) \cos \delta, \\
\tan f & =\tan \delta / \cos (\alpha-185 \pi / 180)
\end{aligned}
$$

( $\alpha$ and $\delta$ must be expressed in radians).

With this choice of coordinates, we can form a regular region, namely, a rectangle, such that it covers most of the SDSS main area. The rectangle is the product of the intervals $-0.7604<s l<0.7934$ and $-0.02269<f<1.1996$, covering a total solid angle that is the product of the respective ranges of $s l$ and $f$, namely, $\Omega=1.554 \cdot 1.222=1.899$ steradians. $^{1}$ This rectangle is displayed in Fig. 1, where the fractions of area do represent fractions of solid angle and hence of galaxy number, because $s l$ and $f$ are equal-area coordinates.

For the radial coordinate $r$, the natural definition is the comoving distance that corresponds to the assumed values of the cosmological parameters. The relation between $r$ and the luminosity distance in Eq. (2.1) is $R=(1+z) r$.

\section{Multifractal analysis}

Let us assume that some mass is distributed in a region of space. The multifractal analysis of this distribution can be carried out in two different ways: either by using a lattice of cells (boxes) covering the region (a method called by Falconer [40] "coarse multifractal analysis") or by using point-centered spheres, where the points span the support of the distribution.

\footnotetext{
${ }^{1}$ The "rectangle" so defined is not a spherical rectangle, because the two opposite sides with constant sl, that is to say, constant latitude $\lambda$, are not great circles and are therefore curved in the intrinsic geometry of the spherical surface.
} 
Harte [41] compares both methods. For a distribution of equal-mass particles, the calculation of the two-point correlation function is equivalent to the calculation of the point-centered second multifractal moment. In fact, the calculation of point-centered statistical moments, or just the second moment, in the form of conditional density, has been the main method of fractal analysis of the SDSS galaxy distribution [31-34]. However, a lattice multifractal analysis is more practical to cope with a large amount of data and avoids the problem of choosing a maximum radius for point-centered spheres. In the analysis of distributions of equal-mass particles, this method boils down to an elaboration of counts-in-cells statistics; but it is also practical for unequal-mass particles. We explain it now.

Given the lattice, fractional statistical moments are defined as

$$
\mathcal{M}_{q}=\sum_{i}\left(\frac{m_{i}}{M}\right)^{q}
$$

where the index $i$ runs over the set of non-empty cells, $m_{i}$ is the total mass of the particles in the cell $i$, while $M=\sum_{i} m_{i}$ is the total mass of all the particles, and $q \in \mathbb{R}$. Some distinguished integral moments are: $\mathcal{M}_{0}$, which is just the number of non-empty cells; $\mathcal{M}_{1}$, normalized to one; and $\mathcal{M}_{2}$, which is related to the two-point correlation function.

In a regular distribution, with a well-defined density everywhere, if we take a sufficiently fine mesh, then the mass contained in any cell is proportional to the cell volume $v$. Therefore, $\mathcal{M}_{q} \sim v^{q-1}$. This does not apply to singular distributions. ${ }^{2}$ But the singularities of a distribution can be such that the $q$-moments are non-trivial power laws of $v$ in the $v \rightarrow 0$ limit. So one can define, for any distribution, the exponents

$$
\tau(q)=3 \lim _{v \rightarrow 0} \frac{\log \mathcal{M}_{q}}{\log v}
$$

provided that the limit exists (for every $q$ ). Such a distribution is called multifractal. For a regular distribution, $\tau(q)=3(q-1)$, whereas for singular distributions the exponents are non-trivial. Of course, the numerical evaluation of the limit in Eq. (3.2) is not feasible and one must be satisfied with finding a constant value of the quotient for sufficiently small $v$, that is to say, in a range of negative values of $\log v$ (a range of small scales). In fact, the exponent is normally defined as the slope of the graph of $\log \mathcal{M}_{q}$ versus $\log v$, and its value is found by numerically fitting that slope.

The standard lattice in multifractal analysis is the Euclidean rectangular and even cubical lattice $[40,41]$. In fact, a cubical lattice is perfectly adapted to the analysis of $N$-body simulations. However, volume-limited galaxy samples are defined in spherical sectors, which makes such lattices inadequate, because they lead to a loss of data. It is preferable to define a rectangular lattice in the coordinates adapted to spherical sectors that have been defined in Sect. 2.2 (which is not a rectangular lattice in Euclidean space). In addition, we require that the cells have identical volume. This can be achieved by dividing the angular-coordinate rectangle, given by the intervals of $s l$ and $f$, into equal area sub-rectangles (like in Ref. [26]), and by also splitting the range of $r$ into intervals with constant $\Delta\left(r^{3}\right)=(r+\Delta r)^{3}-r^{3}$. Such a lattice is not unique and we will further require that the resulting cells are reasonably regular, with aspect ratios not very different from one (Sect. 4.4).

Besides the moment exponents $\tau(q)$, a multifractal is also characterized by its local dimensions. The local dimension $\alpha$ at the point $\boldsymbol{x}$ is the exponent of mass growth from that

\footnotetext{
${ }^{2} \mathrm{~A}$ continuous mass distribution with a well-defined density is said to be absolutely continuous. Although this property may seem natural, the standard methods of randomly generating continuous mass distributions produce strictly singular distributions, namely, distributions with no positive finite density anywhere [42].
} 
point outwards, that is to say, $m(\boldsymbol{x}, r) \sim r^{\alpha(\boldsymbol{x})}$, where $m(\boldsymbol{x}, r)$ is the mass in a ball or box of linear size $r$ centered on $\boldsymbol{x}$. The local dimension measures the "strength" of the singularity: the smaller is $\alpha$, the more divergent is the density at $\boldsymbol{x}$ and the stronger is the singularity. Actually, singularities correspond to $\alpha<3$, that is to say, to a divergent density, whereas points with $\alpha>3$ have vanishing density (if they exist). Every set of points with a given local dimension $\alpha$ constitutes a fractal set with a (Hausdorff) dimension that depends on $\alpha$, denoted by $f(\alpha)$. In terms of $\tau(q)$, the spectrum of local dimensions is given by

$$
\alpha(q)=\tau^{\prime}(q), \quad q \in \mathbb{R},
$$

and the spectrum of fractal dimensions $f(\alpha)$ is given by the Legendre transform

$$
f(\alpha)=q \alpha-\tau(q) .
$$

Standard self-similar multifractals have a typical spectrum of fractal dimensions that spans an interval $\left[\alpha_{\min }, \alpha_{\max }\right]$, is concave (from below), and fulfills $f(\alpha) \leq \alpha[40,41]$. Furthermore, the equality $f(\alpha)=\alpha$ is reached at one point, with $\alpha<3$ and such that $q=1$ in Eq. (3.4) [notice that Eq. (3.2) gives $\tau(1)=0$ ]. The corresponding set of singularities contains the bulk of the mass and is called the "mass concentrate."

As a complement to the multifractal spectrum $f(\alpha)$, it is useful to define the spectrum of Rényi dimensions

$$
D_{q}=\frac{\tau(q)}{q-1},
$$

because they have an information-theoretic meaning [41, 43]. Indeed, they express the powerlaw behavior of the Rényi $q$-entropies of the coarse distribution in the limit of vanishing coarse-graining volume, $v \rightarrow 0$. The dimension of the mass concentrate $\alpha_{1}=f\left(\alpha_{1}\right)=D_{1}$ is associated to the ordinary entropy and is also called the entropy dimension. $D_{0}=-\tau(0)$ is the box-counting dimension of the support of the distribution (let us recall that the support of a mass distribution is the smallest closed set that contains all the mass [40]). $D_{0}$ also coincides with the maximum value of $f(\alpha) . D_{2}=\tau(2)$ is the correlation dimension. For a regular distribution, $\tau(q)=3(q-1)$ and $D_{q}=\alpha=f(\alpha)=3$. For a uniform fractal (a unifractal or monofractal), $\alpha, f(\alpha)$ and $D_{q}$ are also constant but $D_{q}=\alpha=f(\alpha)<3$. In general, $D_{q}$ is a non-increasing function of $q$.

As said above, the convergence to the limit in Eq. (3.2) must take place in a range of small values of $v$. Naturally, $v$ must be small in comparison to the homogeneity volume $v_{0}$, which is the smallest volume such that the mass fluctuations in it are small and approximately Gaussian (assuming that homogeneity holds on sufficiently large scales). For cell sizes $v$ close to $v_{0}$ or larger, the fluctuations tend to vanish and $\mathcal{M}_{q} \approx v^{q-1}$. This relation is an asymptotic equality, provided that the total sample volume is normalized to one. On account of it, we define, for a given cell size $v$, the coarse exponent as

$$
\tau(q)=3 \frac{\log \left(\mathcal{M}_{q} / v_{0}^{q-1}\right)}{\log \left(v / v_{0}\right)} .
$$

In this fraction, both the numerator and denominator vanish when $v$ approaches $v_{0}$ from below (the former approximately and the latter exactly). Their quotient tends to $\tau(q)=$ $3(q-1)$, namely, the form of $\tau$ for regular distributions. The coarse exponent (3.6) depends on both $v$ and $v_{0}$ but must become independent of $v_{0}$ when $v \ll v_{0}$, provided that the limit $v \rightarrow 0$ exists. However, the dependence on $v_{0}$ must not be ignored, because the rate of 
convergence to the limit does depend on the value of $v_{0}$. In particular, if one sets $v_{0}$ to one, namely, the total sample volume, and this volume greatly exceeds the homogeneity volume, the coarse exponents can be so inaccurate that no convergence can be observed and it is not possible to speak of a scaling limit. In other words, if $v_{0}$ is not set correctly, the available range of $v$ may not be long enough for us to obtain reliable values of the functions $\tau(q)$ and $f(\alpha)$. We discuss the choice of $v_{0}$ in Sect. 4.2.

When the cell volume $v$ reaches $v_{0}$, each cell can be considered as an independent realization of the stochastic process that generates the cosmic web structure. The multifractal spectrum $f(\alpha)$ measures the probability of finding mass concentrations of strength $\alpha$ in a realization. This probability is estimated, in a lattice with small $v$, as the number of cells with strength $\alpha$ divided by the number of non-empty cells, approximately, $v^{-f(\alpha) / 3} / v^{-D_{0} / 3}=$ $v^{\left[-f(\alpha)+D_{0}\right] / 3}$. This probability is maximal and close to one when $f(\alpha)$ takes its maximum value $D_{0}$, because most non-empty cells have the corresponding value of $\alpha$. On the contrary, the probability is minimal for $\alpha_{\min }$ or $\alpha_{\max }$, because they normally occur only once. If the sample only occupies the homogeneity volume $\left(v_{0}=1\right)$, then we have just one realization and $f\left(\alpha_{\min }\right)=f\left(\alpha_{\max }\right)=0$, so that the cells with $\alpha_{\min }$ or $\alpha_{\max }$ occur just once. A larger sample $\left(v_{0}<1\right)$ contains more than one realization of the stochastic process. If $\alpha_{\min }$ and $\alpha_{\max }$ still occur only once, then there is less than one cell with $\alpha_{\min }$ or $\alpha_{\max }$ per realization and $f\left(\alpha_{\min }\right)=f\left(\alpha_{\max }\right)<0$; that is to say, there are negative fractal dimensions. This anomaly has been discussed by Mandelbrot [44]. It is linked to the use of coarse multifractal analysis, to the extent that any dependence on $v_{0}$ must disappear as $v \rightarrow 0$. Indeed, when $f(\alpha)<0$, the number of cells with strength $\alpha$ diminishes as $v \rightarrow 0$. In the limit, any set of singularities of strength $\alpha$ with $f(\alpha)<0$ is almost surely empty. Therefore, we can discard this part of the spectrum.

\subsection{Errors in multifractal analysis}

In coarse multifractal analysis, the computation of the coarse exponents (3.6) is subject to errors that increase for small cell volume $v$, with the consequent limitation of the available scale range. The computation of $\mathcal{M}_{q}$ by Eq. (3.1) is subject to errors because the mass $m_{i}$ in each cell is uncertain. This uncertainty is due to the uncertainty in the positions and extensions of galaxies combined with the uncertainty in the galaxy masses, namely, in the available stellar mass estimates of the galaxies. Although the latter type of error does not exist if one assumes that the galaxy masses are equal, this assumption is intrinsically much more erroneous. Adequate methods of error estimation are explained in Sects. 3.2 and 4.5, but it is convenient to consider before some generalities.

The major cause of limitation in the available scale range for a sample lies in the discrete nature of the sample: if there are many galaxies in a cell, the statistical uncertainties in positions or masses tend to compensate each other, whereas the uncertainty is largest in the cells with the smallest number of particles. The effect on the coarse exponent $\tau(q)$ depends on the value of $q$. The form of $\mathcal{M}_{q}$ in Eq. (3.1) shows that, for $q>0$, the errors in the larger values of $m_{i}$ are more important, whereas, for $q<0$, the errors in the smaller values of $m_{i}$ are more important. In consequence, the values of $\tau(q)$ for $q<0$, and hence the values of $f(\alpha)$ for $\alpha>3$ are more affected by discretization errors and are therefore more uncertain. In general, we can say that the geometric features of voids (zones with $\alpha>3$ ) are more difficult to establish, because voids are usually undersampled. Moreover, the uncertainty of $\mathcal{M}_{q}$ for $q<0$ increases with decreasing $v$. The values of $\mathcal{M}_{q}$ for $q>0$ are less uncertain for a given $v$, but their uncertainty also increases with decreasing $v$, as the discretization errors grow. 
In general, the geometry of both clusters and voids is only discernible when they contain sufficient numbers of galaxies.

In the case of equal masses, the variable that rules the discretization errors is the mean number density $n$ of the sample. The discretization length $n^{-1 / 3}$ (length of the cube with one particle on average) is the overall scale for the onset of discretization errors (the smaller scales can be said to belong to the "shot-noise regime" [13]). The structure of cosmic voids, in particular, is lost on scales smaller than the discretization length, while the structure of clusters persists on somewhat smaller scales [22-24]. In fact, it is easy to see that singularities of strength $\alpha$ are sampled down to the length scale $v_{0}^{1 / 3-1 / \alpha} n^{-1 / \alpha}$, where $v_{0}$ is the homogeneity volume. This criterion is only valid for equal masses, but it could be regarded as a heuristic rule for unequal masses. For galaxies in a certain mass range, a higher number density obviously diminishes the discretization errors, but it is not easy to compare the errors for different mass ranges and how they affect different ranges of $\alpha$. We shall see that low-luminosity and therefore low-mass volume-limited samples are generally preferable over the full range of $\alpha$ (Sect. 4.5).

In regard to the actual evaluation of errors, we can estimate the errors of the moments $\mathcal{M}_{q}$ for a given $v$, in terms of the errors in positions and masses (Sects. 3.2 and 4.5), but this does not really tell us how reliably the coarse exponents $\tau(q)$ approach their limit values for $v \rightarrow 0$. In fact, the approach to the $v \rightarrow 0$ limit is easily demonstrated by checking the numerical convergence of coarse exponents. However, convergence can only take place before discretization errors take hold, and this happens at larger values of $v$ for larger $\alpha$ (smaller $q$ ). The maximum scale range for convergence extends from the homogeneity scale $v_{0}^{1 / 3}$ down to the $\alpha$-dependent discretization scale written above, which gives, in the equal-mass case, the scale factor

$$
\frac{v_{0}^{1 / 3}}{v_{0}^{1 / 3-1 / \alpha} n^{-1 / \alpha}}=\left(v_{0} n\right)^{1 / \alpha} .
$$

It is indeed smaller for larger $\alpha$ (and tends to one for $\alpha \rightarrow \infty$ ). The crucial non-dimensional variable is $v_{0} n$, which is the number of particles in a homogeneity volume. This number is very large in recent $N$-body simulations and guarantees good convergence even for $\alpha \gtrsim 4$, as shown in Sect. 3.2. Unfortunately, the situation is much worse in galaxy surveys.

In summary, the estimation of errors in the coarse multifractal spectra for a given sample is less useful than the analysis of convergence of those spectra in the available scale range. This range is limited by the growth of the magnitude of errors for diminishing $v$, which restricts the maximum value of $\alpha$ for which convergence holds. The overall accuracy of our results relies on several consistency checks. First, the results for a given volume-limited galaxy sample must be self-consistent across its available scaling range, which amounts to a proof of convergence to a multifractal limit. Second, the results for different samples must be consistent. Finally, the multifractal geometry of the stellar mass thus obtained, after passing the preceding checks, must be consistent with the multifractal geometry of dark matter and gas derived from $N$-body simulations.

\subsection{Multifractal spectrum from cosmological $N$-body simulations}

The multifractal analysis of LCDM $N$-body simulations shows that a sufficient convergence can be achieved for all values of local dimension $\alpha$ and reveals a typical multifractal spectrum, represented in [22, Fig. 5], [24, Fig. 2], or [26, Fig. 2]. For example, the analysis of the Bolshoi (= Big) simulation [26] gives the multifractal spectrum displayed in Fig. 2. Notice the good convergence of the coarse multifractal spectra, which correspond to the scale of $3.9 \mathrm{Mpc} / h$ (a 


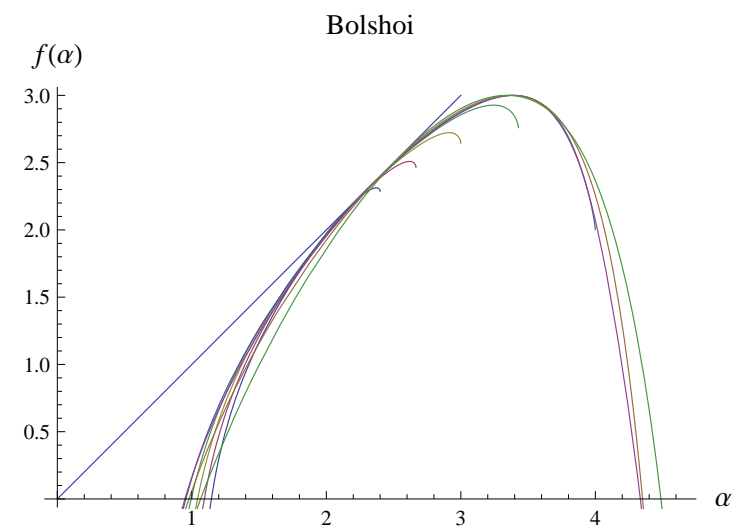

Figure 2. The multifractal spectrum of the present time dark matter distribution in the Bolshoi simulation, as a typical multifractal spectrum of LCDM $N$-body simulations. This graph clearly shows the convergence of coarse multifracta spectra.

fraction $2^{-6}$ of the total box length) and seven subsequently halved scales (a total factor of $\left.2^{7}=128\right)$. But only the larger scales can give the multifractal spectrum in the zone of voids $(\alpha>3)$ and reach the maximum value $f(\alpha)=3$. Essentially the same multifractal spectrum of Fig. 2 is found in other LCDM $N$-body simulations, and, moreover, the analysis of the Mare-Nostrum simulation, which includes gas, shows that the same multifractal spectrum is found for the distribution of gas [24, Fig. 2]. The salient features of the common spectrum are the following.

First of all, it has the typical concave shape that corresponds to a self-similar multifractal $[40,41]$. The maximum value of $f(\alpha)$, equal to the box-counting dimension of the support of the distribution $D_{0}$, is very close to 3 , which is a special value. Notice that $\mathcal{M}_{0}=v^{-1}$ when there are no empty cells in the lattice, yielding, according to Eq. (3.6), $D_{0}=-\tau(0)=3$. However, in the $v \rightarrow 0$ limit, there can be empty cells and what matters is how the number of them grows: if it does not grow at a sufficient rate, then still $D_{0}=3$. Therefore, $D_{0}=3$ occurs either with no voids or with a sequence of voids of sizes that decrease too rapidly. Indeed, what singles out a fractal hierarchy of voids is that it fulfills the Zipf law or that it follows the Pareto distribution [8, 23]. The analysis of LCDM $N$-body simulations, e.g., the Bolshoi simulation in Fig. 2, shows that there are actually no empty cells, starting from $v \lesssim v_{0}$ and well into the scaling range, and the voids that arise on lower scales seem to be due to undersampling. This suggests that the cosmic web has a nonlacunar multifractal geometry, with no totally empty voids [23]. ${ }^{3}$

As regards the mass concentrate, the dimension $\alpha_{1}=f\left(\alpha_{1}\right)$, given by the point of tangency to the diagonal in the graph, seems to be about 2.4, but it cannot be determined precisely. The strongest singularities have $\alpha \simeq 1$. This value, namely, the law of mass growth $m(r) \propto r$, corresponds to the singular isothermal sphere profile or to a filament, in the fully isotropic or extremely anisotropic cases of mass concentrations, respectively. The strongest mass depletions have $\alpha \simeq 4.5$.

The best convergence of coarse multifractal spectra takes place in the $N$-body simulations with the largest values of $v_{0} n$, that is to say, with the best mass resolution. In the

\footnotetext{
${ }^{3}$ This conclusion refers to Mandelbrot's original definition of lacunarity [8] and does not mean that a nonvanishing lacunarity cannot be defined. Indeed, the concept of lacunarity has proved to be subtle and there are various definitions. The notion of nonlacunar fractal, as a fractal set that is everywhere dense, was also introduced by Mandelbrot [8].
} 
Bolshoi simulation, the homogeneity scale is one eighth of the simulation box, which yields an overall scale range $\left(v_{0} n\right)^{1 / 3}=256$. This range is reduced for $\alpha>3$ but is nevertheless sufficient for the largest values of $\alpha$ present: one can observe in Fig. 2 convergence of three coarse multifractal spectra even for $\alpha \simeq 4.5$. For $\alpha<3$, the convergence is still more convincing.

Moreover, it is easy to see that each coarse multifractal spectrum has a negligible error due to errors in particle positions. Particle coordinates are given by floating-point numbers with 23-bit mantissa (excluding the sign bit). The 13 most significant bits are preserved by the coarse graining to the smallest cell used in the Bolshoi simulation (with $0.03 \mathrm{Mpc} / h$ ), leaving a 10-bit precision inside each cell. For larger cells, the precision is higher, of course. Therefore, the relative error in position inside any cell is $<10^{-3}$. The relative error in mass of cells with many particles, which are more important for $q>0$, is proportional to the relative error in positions, and, in fact, the relative error in $\mathcal{M}_{q}$, for $q>0$, is practically equal to the relative error in positions. For $q<0$, cells with few particles can be important in the computation of $\mathcal{M}_{q}$ by Eq. (3.1). However, the sum in that formula can be expressed, for equal-mass particles, as a sum over number of particles per cell, so that each summand is multiplied by the number of cells with a definite number of particles. The error may alter significantly the number of particles in single cells with few particles but will not alter significantly each summand. In fact, the relative error in the number of cells with a definite number of particles is of the order of magnitude of the relative error in position, in any case. This implies that the change in the coarse multifractal spectra induced by errors in particle positions is inappreciable in Fig. 2.

If we consider the good concordance of multifractal spectra for the Bolshoi simulation and other LCDM $N$-body simulations [22, 24], we can say that we have a reliable multifractal spectrum of the LCDM cosmic web. In galaxy samples, the mass resolution is much worse and the errors are considerable, so we should not expect to obtain nearly as accurate a multifractal spectrum, and we must rather compare what we obtain to the $N$-body simulation multifractal spectrum. This is especially true in the zone $\alpha>3$, for which the mass resolution of galaxy samples is hardly sufficient.

\section{Procedure and Results}

Here we describe in detail how we select VL samples in a few redshift intervals, how we determine the value of $v_{0}$, needed for Eq. (3.6), and how we construct coarse lattices appropriate for the selected VL samples. Finally, we calculate the multifractal spectra of these samples.

\subsection{Selection of volume-limited samples}

Previous fractal analyses of SDSS galaxies have either considered several VL samples, in ranges of consecutive absolute magnitudes [31, 33, 34], or focused on a particular sample [32]. In all cases, there has been a bias towards deep VL samples and, therefore, high luminosities, in accord with their common goal of analyzing the transition to homogeneity.

For just finding the multifractal geometry of the mass distribution, namely, the properties of singular mass concentrations and mass depletions, the relevant length scales are necessarily smaller. In the present multifractal analysis of the stellar mass of galaxies, it may seem that we should favor VL samples that contained large fractions of the total stellar mass density. Indeed, such samples represent the mass concentrate set, namely, the set of singularities that contains the bulk of the stellar mass. However, the bulk of the stellar mass 
corresponds to rather bright galaxies and, hence, to moderately deep VL samples. Unfortunately, if we employ these samples, we miss information about voids, which is best obtained from VL samples with fainter and less massive galaxies.

\begin{tabular}{ccccccc}
\hline Redshift & $r(\mathrm{Mpc} / h)$ & $N$ & $V\left(\mathrm{Mpc}^{3} / h^{3}\right)$ & $n=N / V$ & $\rho\left(\mathrm{M}_{\odot} h^{3} / \mathrm{Mpc}^{3}\right)$ & galaxy mass $\left(\mathrm{M}_{\odot}\right)$ \\
\hline \hline$[0.003,0.013]$ & {$[8.99,38.9]$} & 1765 & $3.68 \cdot 10^{4}$ & 0.048 & $8.43 \cdot 10^{6}$ & {$\left[5.8 \cdot 10^{5}, 1.1 \cdot 10^{9}\right]$} \\
\hline$[0.02,0.03]$ & {$[59.7,89.4]$} & 16557 & $3.17 \cdot 10^{5}$ & 0.052 & $2.65 \cdot 10^{8}$ & {$\left[1.7 \cdot 10^{6}, 5.5 \cdot 10^{10}\right]$} \\
\hline$[0.04,0.06]$ & {$[118.9,177.5]$} & 42021 & $2.48 \cdot 10^{6}$ & 0.017 & $1.92 \cdot 10^{8}$ & {$\left[2.1 \cdot 10^{7}, 1.9 \cdot 10^{11}\right]$} \\
\hline
\end{tabular}

Table 1. Characteristics of the three volume limited samples.

Consequently, to construct our VL samples, it is convenient to take first a range of low-redshift galaxies, that is to say, faint galaxies, and then proceed to deeper samples. In fact, we can do with just three VL samples, chosen as follows: VLS1 with $z \in[0.003,0.013]$, VLS2 with $z \in[0.02,0.03]$, and VLS3 with $z \in[0.04,0.06]$ (see Table 1). The first one is actually the most useful one, because not only is it useful for the study of voids (the zone $\alpha>3$ ) but also, as it turns out, for the study of clusters (the zone $\alpha<3$ ). The reason is that the information on clusters can be obtained not only from the more massive galaxies, which are to be found mainly in clusters, but also from the patterns of clustering of the less massive galaxies. We indeed find that the multifractal spectra obtained from the three VL samples in the zone $\alpha<3$ are practically equal (Sect. 4.5).

\subsection{Homogeneity scale}

To implement the procedure of coarse multifractal analysis described in Sect. 3, we need to compute the $q$-moments $\mathcal{M}_{q}$ from the set of cell masses $m_{i}$, and then compute the coarse exponents $\tau(q)$, according to Eq. (3.6), and do it for several lattices, with decreasing cell volumes $v$. To compute the coarse exponents, we need first to calculate the homogeneity volume $v_{0}$. We encounter here, of course, an old problem: the determination of the scale of homogeneity of the universe. The SDSS data have been employed for this purpose, with various results [31-34]. In fact, the "scale of homogeneity" is a loose concept and, as such, is bound to be defined in different ways, which produce different results, even if applied to the same data. A practical definition can be given in terms of the normalized second moment $\mu_{2}(v)=\left\langle\rho_{v}^{2}\right\rangle=\mathcal{M}_{2}(v) / v$, where $\rho_{v}$ is the normalized coarse-grained density and the last equality assumes that there are no empty cells in the lattice. Homogeneity is defined by $\mu_{2}=1$, but this value is only approached asymptotically for large $v$ (or as $v$ approaches the full sample volume). The difference $\mu_{2}-1=\left\langle\left(\rho_{v}-1\right)^{2}\right\rangle$ measures the mass variance in the volume $v$ and can be used, in general, to determine the regime of galaxy clustering [13]: in the homogeneous regime, the coarse-grained density is Gaussian with a small or nearly vanishing variance.

The criterion for choosing $v_{0}$ in Refs. $[24,26]$ actually was that $\mu_{2}\left(v_{0}\right)=1.1$, that is to say, a variance of $10 \%$. This criterion is simple but is as arbitrary as any other, of course, because one can as well demand smaller variances, say $5 \%, 1 \%$ or less, hence considerably increasing the value of $v_{0}$. Indeed, the claims that homogeneity has not been found yet in galaxy catalogs are surely due to imposing too strict criteria for homogeneity. ${ }^{4}$ Without considering any specific property of the mass distribution, in addition to its having, on small

\footnotetext{
${ }^{4}$ In fact, what is claimed by some authors is that there are signs of inhomogeneity on very large scales $[31,33,34]$. However, certain signs of inhomogeneity that may look like structures can be observed in a mass distribution with small mass-variance, for example, in a fluid in a critical state [13].
} 

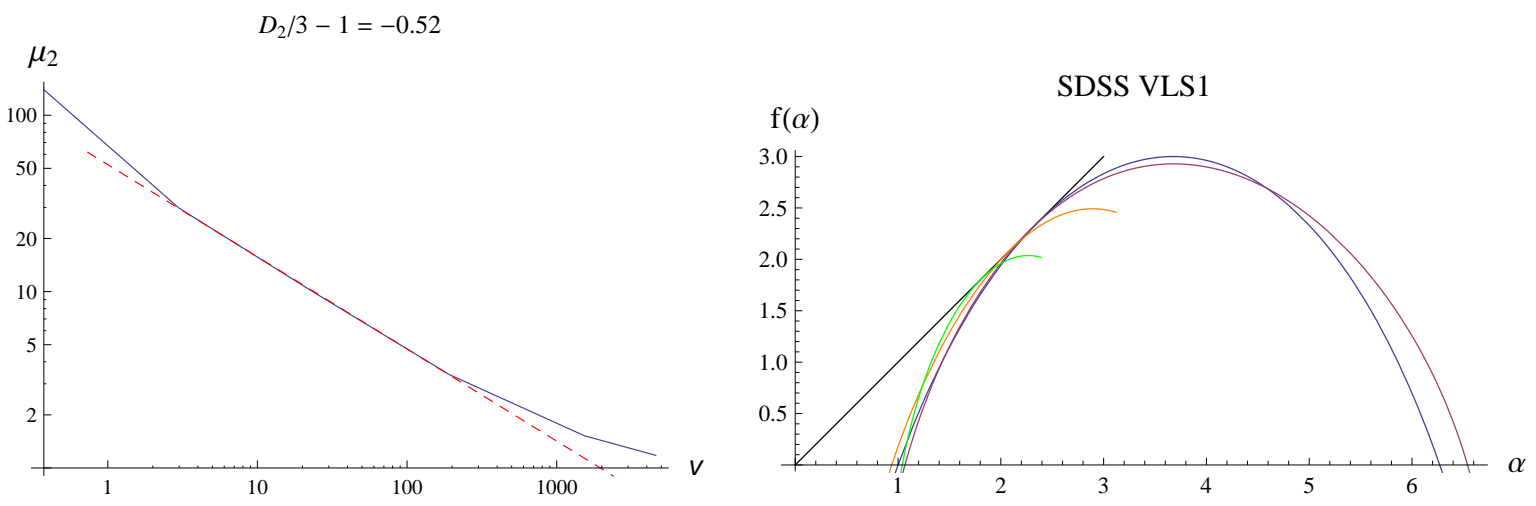

Figure 3. Results of the analysis of sample VLS1: On the left-hand side, moment $\mu_{2}(v)(v$ in $\left.\mathrm{Mpc}^{3} / h^{3}\right)$, with a fit of the scaling part $\propto v^{D_{2} / 3-1}$. On the right-hand side, multifractal spectrum for coarse-graining volumes $v=2.99,23.9,191 ., 1.53 \cdot 10^{3}(\mathrm{Mpc} / h)^{3}$.

scales, large values of $\mu_{2}$, that is to say, its being strongly non-Gaussian, one cannot propose a definite criterion and one is confined to speculating about what is a sufficiently Gaussian distribution. However, the scaling of $\mathcal{M}_{q}(v)$ and, in particular, of $\mathcal{M}_{2}(v)$ allows us to define a more precise scale of transition to homogeneity, namely, the scale of crossover from the multifractal scaling with non-trivial values of $D_{q}$ to the homogeneous scaling with $D_{q}=3$ for all $q$. It has been shown, in some cases, that this criterion roughly agrees with the $10 \%$ mass variance criterion $[24,26]$. Therefore, we also examine here the scaling of $\mathcal{M}_{2}(v)$. To be precise, we examine the crossover from the scaling $\mu_{2} \sim v^{D_{2} / 3-1}$ on middle scales to the exact value $\mu_{2}=1$ on very large scales. The value of $v_{0}$ obtained in this way is sufficient for our purpose, namely, for its use in Eq. (3.6), and we do not need to consider subtle issues about the concept of homogeneity (see Ref. [13]).

\subsection{Values of $v_{0}$ for our volume-limited samples}

The calculation of the scale of transition to homogeneity as the scale of crossover from a multifractal scaling to the homogeneous scaling in, for example, VLS1 can be seen in the $\log$-log plot of the corresponding $\mu_{2}(v)$, displayed in Fig. 3. This plot contains a fit of the scaling $\mu_{2} \sim v^{D_{2} / 3-1}$, in the interval $v \in[3,190] \mathrm{Mpc}^{3} / h^{3}$, with the result $D_{2}=1.44$. Of course, the fitting line and the consequent value of $D_{2}$ change if we change the interval of $v$, but one must choose an interval that yields a good fit (with small errors). The scale of crossover can be taken as the value of $v$ at the crossing of the fitting line (for the fractal scaling) and the line $\mu_{2}=1$ (for homogeneity), which yields $v_{0}=2000 \mathrm{Mpc}^{3} / h^{3}$. However, the corresponding value of $\mu_{2}$ is somewhat high, that is to say, hardly compatible with a Gaussian distribution. So we take a larger value of $v_{0}$, such that the magnitude of $\mu_{2}-1$ is somewhat smaller. Notice that we can as well slightly increase the upper end of the interval of $v$ to fit, which will lower the absolute value of the slope and therefore increase the value of $v$ at the crossing point (and will also increase $D_{2}$ ). Relying on these arguments, we take, for this sample, $v_{0}=4600 \mathrm{Mpc}^{3} / h^{3}$ (the cell size in a $2 \times 2 \times 2$ lattice, see Sect. 4.4).

To $v_{0}=4600(\mathrm{Mpc} / h)^{3}$ corresponds the homogeneity length scale $4600^{1 / 3} \mathrm{Mpc} / h=17$ $\mathrm{Mpc} / h$, which is in reasonable agreement with the results from $N$-body simulations $[24,26]$, although it is smaller than other values for SDSS galaxies [31-34]. These values are obtained with different criteria, which are probably too strict, at least, for our purpose. Nevertheless, 
we find that the appropriate homogeneity scales for VLS2 and VLS3 are a little larger, reaching $25 \mathrm{Mpc} / h$ for VLS3. This value is practically equivalent to the value of $30 \mathrm{Mpc} / h$ obtained by Verevkin et al [33], also for the SDSS-DR7 (although they counter that the uniform regime on larger scales still has some sort of inhomogeneities).

\subsection{Coarsening the volume-limited samples}

As explained in Sect. 3, our multifractal analysis is carried out in lattices of equal-volume cells that are formed by a particular Cartesian product. This product results from multiplying an angular-coordinate lattice, with the intervals of $s l$ and $f$ divided into equal subintervals, by the interval of $r$ divided into subintervals with constant increment of $r^{3}$. Additionally, we want the resulting cells to be reasonable regular, with aspect ratios not very different from one.

For each sample, we must prepare a set of diminishing meshes. To achieve good aspect ratios, we need to adapt the set of meshes to the particular interval of $r$ of each sample. For the sake of computational simplicity, once the initial coarse mesh is chosen for a sample, we generate a sequence of finer meshes by using binary division of subintervals. Given the ranges of $s l$ and $f$, respectively, 1.55 and 1.22, an initial $4 \times 3$ angular lattice generates angular cells with aspect ratio close to one (see the right part of Fig. 1). The interval of $r$ depends on the sample, but the shape of the spherical sector for a sample is only determined by the ratio of the upper to the lower limits of $r$. This ratio is almost the same for VLS2 and VLS3, and it is such that for all the three samples the length of the radial interval is smaller than the length of the angular intervals. We find that initial coarse meshes of $4 \times 3 \times 2$ and $4 \times 3 \times 1$ yield acceptable aspect ratios for, respectively, VLS1 and VLS2 (or VLS3). The volume of the cells in the initial 24-cell lattice for VLS1 is actually somewhat small, so we add in this case a coarser mesh, namely, a $2 \times 2 \times 2$ mesh, to probe larger scales and, in particular, to find the transition to homogeneity.

\subsection{Multifractal spectrum}

Several coarse multifractal spectra of VLS1, computed with Eq. (3.6) and $v_{0}=4600 \mathrm{Mpc}^{3} / h^{3}$, are displayed in Fig. 3. The agreement with the spectrum obtained from $N$-body simulations (Sect. 3.2 and Fig. 2) is very convincing in the zone $\alpha<3$, corresponding to singularities and therefore galaxy clusters. Indeed, the dimension of the mass concentrate, given by the points of tangency to the diagonal, is between 2 and 2.6 and is probably about 2.4; and the strongest singularities also have $\alpha \simeq 1$. For $\alpha \gtrsim 3$, we have convergence of only two coarse multifractal spectra, but they show anyhow that the maximum value of $f(\alpha)$, namely, the box-counting dimension of the distribution's support, is very close to 3. However, the fact that all (or almost all) cells are non-empty for just two scales, which are not well below $v_{0}$, is not a fully convincing proof of non-lacunarity. Anyway, the concordance between this galaxy sample and the results of $N$-body simulations is remarkable, because the mass resolution of galaxies is generally much worse and, furthermore, galaxy data are subject to considerable errors (see below). Unfortunately, the concordance breaks down for $\alpha>4$ and, in particular, the largest value of $\alpha$ is notably larger in Fig. 3 than in Fig. 2.

These results are supported by the estimation of errors in the VLS1 coarse spectra, due to errors in galaxy positions and masses. The errors in angular and radial coordinates have different origin: the uncertainty in angular positions is mainly due to the size of galaxies, whereas the uncertainty in distance is mainly due to the uncertainty in the Hubble law caused by peculiar velocities. The size of the type of galaxies in VLS1 can be calculated in terms of galaxy mass according to $R(\mathrm{kpc})=0.1\left(M / M_{\odot}\right)^{0.14}[45]$. This yields a maximum radius $<2$ 

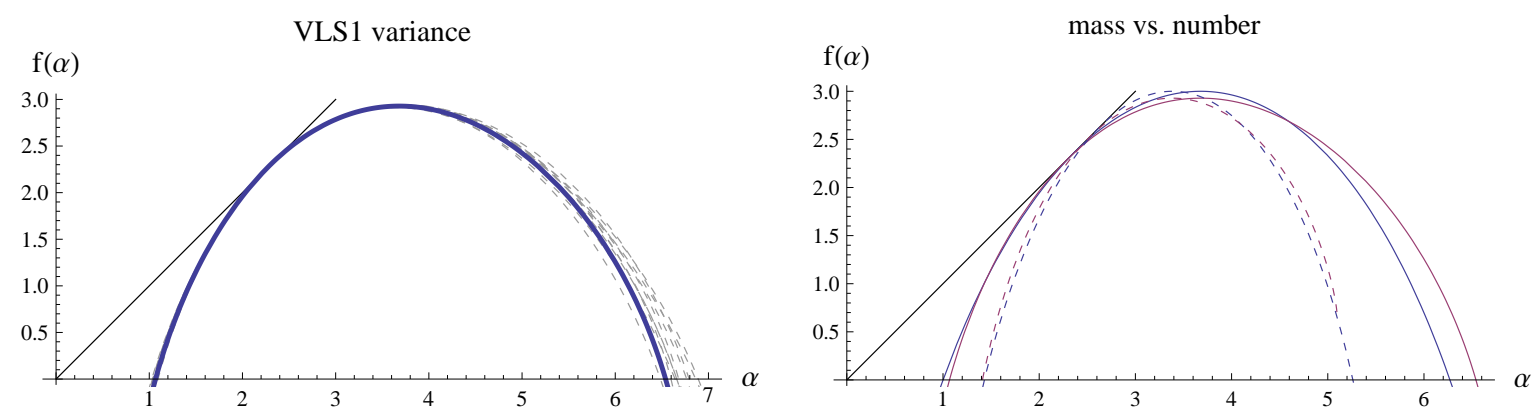

Figure 4. Errors in multifractal spectra: (Left) Variance in the spectrum of VLS1 at $v=191$ $(\mathrm{Mpc} / h)^{3}$ due to errors in galaxy positions and masses (ten variant spectra, in dashed lines). (Right) Effect of suppressing galaxy masses in VLS1 at $v=191 ., 1.53 \cdot 10^{3}(\mathrm{Mpc} / h)^{3}$ (in dashed lines).

$\mathrm{kpc}$, which is negligible in comparison with the sizes of the coarse-graining cells, larger than $1 \mathrm{Mpc} / h$ (the error is just a bit larger than the error in the Bolshoi simulation, Sect. 3.2). As regards radial coordinates, peculiar velocities actually destroy the Hubble flow on small scales and, hence, the determination of distance by redshift. However, the local Hubble flow is "cold" and the dispersion of peculiar velocities is as low as $30 \mathrm{~km} / \mathrm{s}$ [46]. To be on the safe side, we take a dispersion of $50 \mathrm{~km} / \mathrm{s}$. Therefore, we assume, for the error in distance, that redshifts have normal (Gaussian) errors with dispersion $\sigma_{z}=1 / 6000$. Notice that this error is certainly non-negligible at the lower cut in redshift, $z=0.003$.

The error in stellar mass estimates is such that $\log _{10} M \pm 0.3$ spans the $95 \%$ confidence interval [27, Fig. 15]. Given that a $2 \sigma$ Gaussian deviation interval comprises $95 \%$ of probability, we can assume for $M$ a lognormal distribution with $\sigma_{M}=(\ln 10) 0.3 / 2=0.3454$ (using natural logarithms). This distribution is quite skewed, because the dispersion is considerable: indeed, the $95 \%$ confidence interval amounts to a factor of 4 in mass. Notice that the uncertainty in mass is much larger than the uncertainty in position.

Once we have the $z$ and $M$ distributions, we can generate a number of random alternatives to our initial sample, before the construction of the VL samples. From those alternative initial samples, we construct the corresponding variants of VLS1, following the same procedure followed for VLS1 itself. Hence, we compute the corresponding coarse multifractal spectra. We have done so for ten alternative initial samples, focusing on the most relevant VLS1 multifractal spectrum, namely, the spectrum of VLS1 at $v=191(\mathrm{Mpc} / h)^{3}$, which is within the scaling range yet is reasonable in the void zone $(\alpha>3)$, as seen in Fig. 3 . The variance in this coarse multifractal spectrum is displayed in the left-hand side of Fig. 4 . The graph shows that the error is negligible in the cluster zone $(\alpha<3)$, and even so up to the maximum of $f(\alpha)$. The error grows for $\alpha>4$ and is considerable at the end of the spectrum, in accord with the insufficient mass resolution in that zone, as manifested by the convergence of only two coarse spectra.

One further "error check" is carried out by considering the effect of making all the galaxy masses equal. Such a drastic simplification is beyond any magnitude of error in stellar mass estimation but is relevant for a comparison with the approach that only considers the number of galaxies. Employing two relevant coarse multifractal spectra of VLS1, namely, the ones for $v=191$ and $1.53 \cdot 10^{3}(\mathrm{Mpc} / h)^{3}$, we see in the right-hand side of Fig. 4 the effect of suppressing mass information. Remarkably, the range of $\alpha$ shrinks, more at the right end, belonging to voids, than at the left end, belonging to clusters. But the effect is notable in both cases and actually is more important if $\alpha<3$, because this is the more reliable part of 

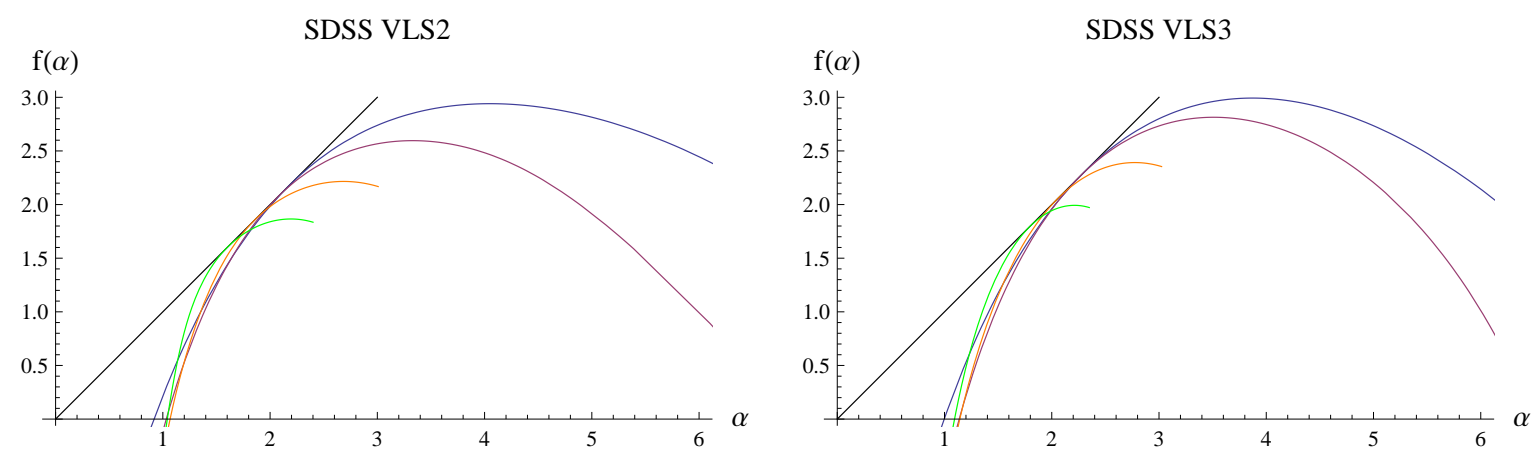

Figure 5. Results of the analysis of samples VLS2 and VLS3.

the spectrum. In fact, by suppressing mass information, we ruin the concordance with the spectrum obtained from $N$-body simulations in that zone. The magnitude of the effect of suppressing masses is undoubtedly due to the broad range of masses (see Table 1).

We now proceed to the analysis of deeper VL samples, namely, VLS2 and VLS3. It does not provide new information: the results for clusters are nearly the same as before but the results for voids are definitely worse (Fig. 5). In fact, the absence of any convergence of coarse multifractal spectra in the zone $\alpha>3$ shows that these deeper VL samples are useless for the study of voids: the voids in them are due to undersampling. Coleman and Pietronero [9], in their multifractal analysis of the CfA catalog, already noticed that $\tau(q)$ cannot be obtained for negative $q$ because $q<0$ corresponds to the lowest masses, which are not well represented. Indeed, $\tau(q)$ for $q<0$ yields the part of $f(\alpha)$ beyond its maximum. At any rate, the part of $f(\alpha)$ up to its maximum that Coleman and Pietronero [9] calculate does not agree with our results: they obtain that $\alpha_{\min }=0.65$ and that the maximum value of $f$ is equal to 1.5 , in contrast with our values $\alpha_{\min }=1$ and maximum of $f$ equal to 3 . The multifractal analyses of galactic catalogs by other authors were based on the galaxy number density but obtained results similar to Coleman and Pietronero's, that is to say, obtained that $\alpha_{\min }$ is smaller than one and that the maximum of $f$ is quite smaller than three. The use of better data and the reasonable agreement with the results of $N$-body simulations in our analysis make it more reliable.

Let us compare our multifractal analysis with the recent analysis of the SDSS-DR7 by Chacón-Cardona et al [34]. This analysis is made in terms of Rényi dimensions $D_{q}$, which provide equivalent information to the multifractal spectrum $f(\alpha)$, from a mathematical viewpoint (Sect. 3). However, Chacón-Cardona et al select deep VL samples, consider a distribution of dark matter halos associated to SSDS-DR7 galaxies instead of the galaxies themselves, and do not take into account the galaxy masses. Moreover, their definition of $D_{q}$ as a derivative with respect to scale is different from the standard definition of fractal dimension adopted here, in terms of the limit for vanishing scale [40, 41]. In consequence, it is difficult to compare directly the present analysis with the analysis by Chacón-Cardona et al [34]. Naturally, the first problem is to compare their results for $D_{q}$ with the present results for $f(\alpha)$. A few partial comparisons are simple to make: for example, the value of the maximum of $f(\alpha)$ must be equal to $D_{0}$. However, while we have found, with confidence, that the maximum of $f(\alpha)$ is 3, no definite value of $D_{0}$ can be deduced from [34, Fig. 4].

At any rate, we believe that the knowledge of the multifractal spectrum $f(\alpha)$ of the large scale stellar-mass distribution is more directly useful than the knowledge of Rényi dimensions, in general, because it allows us to deduce several consequences, which we discuss next. 


\section{Conclusions and discussion}

We have calculated the multifractal spectrum of the large scale stellar-mass distribution, employing the SDSS-DR7, in an effort to determine the geometry of the baryonic cosmic web and see how it relates to the geometry of the dark matter cosmic web. Of course, the stellar mass is only a fraction of the total baryonic mass and, furthermore, the SDSS data do not contain all the stellar mass, because of the cuts in apparent magnitudes of galaxies (and the additional cuts in VL samples). Nevertheless, the information obtained is representative. A different type of information on the distribution of baryonic mass is provided by $N$-body simulations containing baryonic gas. From one of them, we can conclude that the fractal geometry of the distributions of gas and dark matter is the same. Assuming this identity, the question that we are addressing is whether or not the fractal geometry of the distribution of the visible stellar mass coincides with that already known common geometry.

Unfortunately, even the rich SDSS data are insufficient for fully determining the multifractal geometry of the stellar mass distribution. At any rate, we can assert the overall consistency of the stellar mass multifractal spectrum, namely, the internal consistency of our multifractal analysis of the SDSS data and, furthermore, its consistency with the multifractal analysis of LCDM $N$-body simulations. While the internal consistency is clear in the case of mass concentrations (clusters), it is however questionable in the case of mass depletions (voids), since we only have convergence, at best, of two coarse multifractal spectra (the minimum number to speak of convergence) and only for one sample. Therefore, we discuss the cluster $(\alpha<3)$ and void $(\alpha>3)$ cases separately, beginning with the former.

The value $\alpha_{\min }=1$, common to our analysis of the SDSS-DR7 and $N$-body simulations, corresponds to the singular isothermal sphere profile, the standard profile of the outskirts of individual galaxies, and also corresponds to a filament, a basic element of the cosmic web. More in general, it is a natural lower limit, because the gravitational potential diverges at a point on which mass concentrates with $\alpha<1$. Such a mass concentration would not only have a divergent mass density, which may not be physically forbidden, but would also involve a divergent (negative) gravitational energy, which is certainly forbidden. Of course, the gravitational energy would not actually become infinite, because the cosmic-web mass distribution is not valid down to infinitely small scales and the scaling law $m(r) \sim r^{\alpha}$ must change as $r \rightarrow 0$, but mass concentrations with $\alpha<1$ are anyhow linked to excessive energy dissipation and, therefore, must be unlikely to appear. Let us remark that some mass concentrations with $\alpha<1$ do appear in our analysis, but they have $f(\alpha)<0$ and are not displayed in Fig. 3 or Fig. 5. Moreover, $\alpha<1$ values with $f(\alpha)<0$ also appear in the analysis of $N$-body simulations [24]. However, mass concentrations with $f(\alpha)<0$ tend to disappear as $v \rightarrow 0$, as explained in Sect. 3, and this is indeed what we observe.

In this regard, let us notice that the adhesion model predicts that knots are widespread in the cosmic-web, and these knots are points with finite mass, that is to say, singularities of maximum strength, $\alpha=0$. They do not seem to be present in the real cosmic web. The reason for this discrepancy is, of course, that the Zeldovich approximation can only describe gravitational dynamics on the larger scales and is unable to describe gravitational collapse with strong energy dissipation.

Singularities with $\alpha$ close to one are very significant energy-wise, but the total mass that they contain is insignificant. The bulk of the mass concentrates on a set of singularities with dimension $\alpha_{1}=f\left(\alpha_{1}\right)=D_{1} \simeq 2.4$. This is a remarkably high value, especially, when we compare it with the results of older multifractal analyses of the galaxy distribution (with or without galaxy masses), which obtain a maximum of $f(\alpha)$ that is about 2: but the dimension 
of the mass concentrate must be lower than the maximum dimension, of course. As regards the cosmic-web morphology, one could be tempted to conclude that such a high dimension of the mass concentrate favors cosmic sheets over filaments. However, such conclusion would not be warranted at all, because the fractal dimension does not directly give information on morphology and one should employ instead the topological dimension and measures of texture $[8]$.

Regarding void regions, formed by points with $\alpha>3$, the main conclusion is that the maximum value of $f(\alpha)$, which gives the (box-counting) dimension of the support of the distribution, is very close to 3 (and is at $\alpha \simeq 3.5$ ). The dimension 3 corresponds to nonfractal support and suggests that the cosmic web is a non-lacunar multifractal. To confirm it, a specific study of voids is necessary. From the study of voids in Ref. [23] and from the multifractal analysis of $\mathrm{N}$-body simulations, it can be concluded with confidence that the geometry of the dark matter or the baryonic gas is non-lacunar, while the present analysis of the stellar mass distribution is not as conclusive. Voids can be perceived in the galaxy distribution (e.g., in the right-hand side of Fig. 1) but they are, presumably, an effect of undersampling. This effect can combine with the existence of regions with very low baryonic density and therefore very few stars. It is to be remarked that the cosmic web generated by the adhesion model, although somewhat different from the real cosmic web, is also an example of the peculiar geometry of non-lacunar fractals [23].

For strong mass depletions, with $\alpha>4$, the result of our analysis of the SDSS differs from the result of analyses of $N$-body simulations. The latter shows a rather sharp decline of the dimension of point sets with $\alpha>4$ whereas the decline shown by the SDSS analysis is more progressive. We must caution that the SDSS data are hardly sufficient to be confident about this conclusion. In this regard, we must wait for more information, from future surveys able to detect much fainter galaxies. Other types of data can also be employed; for example, data on the intergalactic matter.

Finally, we can compare the value $D_{2}=1.44$, obtained by fitting the scaling $\mu_{2} \sim$ $v^{D_{2} / 3-1}$ in VLS1, with other values of the correlation dimension. Sylos Labini et al [31] and Verevkin et al [33] obtain $D_{2}=2$ (or higher) from the SDSS data, employing the DR4 and DR7, respectively. However, smaller values, obtained from various samples, appear in the literature [14]. We must caution that the often calculated and discussed correlation dimension is always the one that corresponds to the galaxy position correlation function, whereas our value of $D_{2}$ corresponds instead to the correlation function of the stellar mass distribution.

\section{Acknowledgments}

I thank C.A. Chacón-Cardona for correspondence and for the SDSS-DR7 file.

\section{References}

[1] Ya.B. Zeldovich, Gravitational instability: An approximate theory for large density perturbations, Astron. \& Astrophys. 5 (1970) 84-89.

[2] S.N. Gurbatov, A.I. Saichev and S.F. Shandarin, Large-scale structure of the Universe. The Zeldovich approximation and the adhesion model, Phys. Usp. 55 (2012) 223-249.

[3] J. Einasto, M. Jõeveer and E. Saar, Structure of superclusters and supercluster formation, MNRAS 193 (1980) 353-375.

[4] M.J. Geller and J.P. Huchra, Mapping the Universe, Science 246 (1989) 897-903. 
[5] A.A. Klypin and S.F. Shandarin, Three-dimensional numerical model of the formation of large-scale structure in the Universe, MNRAS 204 (1983) 891-907.

[6] D. H. Weinberg and J. E. Gunn, Large-scale Structure and the Adhesion Approximation, MNRAS 247 (1990) 260-286.

[7] L. Kofman, D. Pogosyan, S.F. Shandarin and A.L. Melott, Coherent structures in the universe and the adhesion model, The Astrophysical Journal 393 (1992) 437-449.

[8] B.B. Mandelbrot, The fractal geometry of nature (rev. ed. of: Fractals, 1977), W.H. Freeman and Company (1983).

[9] P.H. Coleman and L. Pietronero, The fractal structure of the Universe, Phys. Rep. 213 (1992) 311-389.

[10] P.J.E. Peebles, Principles of Physical Cosmology, Princeton University Press (1993).

[11] S. Borgani, Scaling in the Universe, Phys. Rep. 251 (1995) 1-152.

[12] F. Sylos Labini, M. Montuori and L. Pietronero, Scale invariance of galaxy clustering, Phys. Rep. 293 (1998) 61-226.

[13] J. Gaite, A. Domínguez and J. Pérez-Mercader, The fractal distribution of galaxies and the transition to homogeneity, Astrophys. J. 522 (1999) L5-L8.

[14] B.J. Jones, V. Martínez, E. Saar and V. Trimble, Scaling laws in the distribution of galaxies, Rev. Mod. Phys. 76 (2004) 1211-1266.

[15] L. Pietronero, The fractal structure of the universe: Correlations of galaxies and clusters and the average mass density, Physica A 144 (1987) 257-284.

[16] B.J. Jones, V. Martínez, E. Saar and J. Einasto, Multifractal description of the large-scale structure of the universe, Astrophys. J. 332 (1988) L1-L5.

[17] R. Balian and R. Schaeffer, Galaxies: Fractal dimensions, counts in cells, and correlations, Astrophys. J. 335 (1988) L43-L46.

[18] R. Valdarnini, S. Borgani and A. Provenzale, Multifractal properties of cosmological N-body simulations, Astrophys. J. 394 (1992) 422-441.

[19] S. Colombi, F.R. Bouchet and R. Schaeffer, Multifractal analysis of a cold dark matter universe, Astron. 83 Astrophys. 263 (1992) 1.

[20] G. Yepes, R. Domínguez-Tenreiro and H.P.M. Couchman, The scaling analysis as a tool to compare $N$-body simulations with observations - Application to a low-bias cold dark matter model, Astrophys. J. 401 (1992) 40-48.

[21] J. Gaite, The fractal distribution of haloes, Europhys. Lett. 71 (2005) 332-338.

[22] J. Gaite, Halos and voids in a multifractal model of cosmic structure, Astrophys. J. 658 (2007) 11-24.

[23] J. Gaite, Statistics and geometry of cosmic voids, JCAP 11 (2009) 004.

[24] J. Gaite, Fractal analysis of the dark matter and gas distributions in the Mare-Nostrum universe, JCAP 3 (2010) 006.

[25] C.A. Chacón-Cardona and R.A. Casas-Miranda, Millennium simulation dark matter haloes: multifractal and lacunarity analysis and the transition to homogeneity, MNRAS 427 (2012) 2613-2624.

[26] J. Gaite, Smooth halos in the cosmic web, JCAP 4 (2015) 020.

[27] G. Kauffmann et al, Stellar Masses and Star Formation Histories for $10^{5}$ Galaxies from the Sloan Digital Sky Survey, MNRAS 341 (2003) 33-53.

[28] M.R. Blanton and S. Roweis. K-Corrections and Filter Transformations in the Ultraviolet, Optical, and Near-Infrared, AJ 133 (2007) 734-754. 
[29] M.R. Blanton et al, New York University Value-Added Galaxy Catalog: A Galaxy Catalog Based on New Public Surveys, AJ 129 (2005) 2562-2578.

NYU Value-Added Galaxy Catalog web page.

[30] E. Tago et al, Groups of galaxies in the SDSS Data Release 7. Flux- and volume-limited samples, Astron. Astrophys. 514 (2010) A102.

[31] F Sylos Labini, NL Vasilyev and YV Baryshev, Power law correlations in galaxy distribution and finite volume effects from the Sloan Digital Sky Survey Data Release Four, Astron. Astrophys. 465 (2007) 23-33.

[32] P. Sarkar, J. Yadav, B Pandey and S. Bharadwaj, The scale of homogeneity of the galaxy distribution in SDSS DR6, MNRAS 399 (2009) L128-31.

[33] A.O. Verevkin, Y.L. Bukhmastova and Y.V. Baryshev, The non-uniform distribution of galaxies from data of the SDSS DR7 survey, Astron Rep 55 (2011) 324-40.

[34] C.A. Chacón-Cardona, R.A. Casas-Miranda, J.C. Muñoz-Cuartas, Multi-fractal analysis and lacunarity spectrum of the dark matter haloes in the SDSS-DR\%, Chaos, Solitons and Fractals 82 (2016) 22-33.

[35] K.N. Abazajian et al, The Seventh Data Release of the Sloan Digital Sky Survey, ApJS 182 (2009) 543-558.

[36] M.R. Blanton et al, The Luminosity Function of Galaxies in SDSS Commissioning Data, AJ 121 (2001) 2358-2380.

[37] I. Chilingarian, A.-L. Melchior and I. Zolotukhin, Analytical approximations of K-corrections in optical and near-infrared bands, MNRAS 405 (2010) 1409.

[38] E.W. Weisstein, Cylindrical Equal-Area Projection, from MathWorld - A Wolfram Web Resource [http://mathworld.wolfram.com/CylindricalEqual-AreaProjection.html].

[39] C. Stoughton et al, Sloan Digital Sky Survey: Early Data Release, The Astronomical Journal 123 (2002) 485-548.

[40] K. Falconer, Fractal Geometry (Second Edition), John Wiley and Sons, Chichester, UK, (2003), Chapter 17.

[41] D. Harte, Multifractals. Theory and applications, Chapman \& Hall/CRC, Boca Raton, Fda. (2001).

[42] M. Monticino, How to Construct a Random Probability Measure, International Statistical Review 69 (2001) 153-167.

[43] A. Rényi, Calcul des probabilités, Dunod, Paris (1966).

[44] B.B. Mandelbrot, Negative fractal dimensions and multifractals, Physica A 163 (1990) 306-315.

[45] S. Shen et al, The size distribution of galaxies in the Sloan Digital Sky Survey, MNRAS 343 (2003) 978-994.

[46] I.D. Karachentsev, O.G. Kashibadze, D.I. Makarov and R.B. Tully, The Hubble flow around the Local Group, MNRAS 393 (2009) 1265-1274. 